\title{
O USO INDISCRIMINADO DE CLONAZEPAM E A IMPORTÂNCIA DA ASSISTÊNCIA DE ENFERMAGEM
}

\section{THE INDISCRIMINATE USE OF CLONAZEPAM AND THE IMPORTANCE OF NURSING CARE}

\author{
Raíssa Cristine Amaral Costa \\ Acadêmica do $9^{\circ}$ Período do Curso de Enfermagem da Universidade Presidente Antônio \\ Carlos UNIPAC Teófilo Otoni-MG - E-mail: ra.maral@outlook.com
}

Daniel de Azevedo Teixeira

Farmacêutico-Bioquímico Orientador. Doutor em Biocombustíveis-UFVJM. Professoradjunto da universidade Presidente Antônio Carlos UNIPAC Teófilo Otoni - MG - E-mail: danielteixeira@unipacto.com.br

Martha Honorato da Silva

Enfermeira. Coorientadora. Formada pela Universidade Unificada DOCTUM de Teófilo Otoni - MG - Especialista em Urgência e Emergência e saúde da família. Professora-adjunta da universidade Presidente Antônio Carlos UNIPAC Teófilo Otoni - MG. E-mail: marthahonorato@gmail.com

\begin{abstract}
Resumo
O uso de clonazepam em todo Brasil tem sido fator de preocupação na área de saúde pública e em vários outros aspectos, devido à grande quantidade de dispensação. Este estudo tem como objetivo discutir o uso indiscriminado de clonazepam e demonstrar a importância da assistência de enfermagem, trata-se de uma pesquisa documental com fundamentação bibliográfica, feita por meio da obtenção de dados de dispensação do medicamento disponibilizado pela Agência Nacional de Vigilância Sanitária (ANVISA) e um estudo feito em artigos e bibliografias para que seja possível identificar como os profissionais de enfermagem podem intervir para que esse problema seja amenizado ou resolvido. $\mathrm{O}$ uso do Clonazepam vem aumentando nos últimos anos, mas apesar da sua segurança pelo seu alto índice terapêutico, seu baixo risco de toxicidade e raros casos de overdose, se usado por tempo prolongado pode gerar problemas como tolerância, dependência e até mesmo crises de abstinência durante a retirada do medicamento. Entende-se, que a busca por medicamentos que aliviem os sintomas de problemas de saúde mental e a falta de informações suficientes tem gerado um uso indiscriminado, trazendo como principal consequência a dependência. Diante disso, acredita-se que o enfermeiro deve ter conhecimento e preparo suficiente para atuar diminuindo o uso de clonazepam, junto com a esquipe multiprofissional, pois o conhecimento sobre a ação desse medicamento como causador de dependência química, bem como o planejamento e implantação de estratégias, podem contribuir significativamente prevendo e minimizando efeitos danosos.
\end{abstract}


Palavras-Chave: Clonazepam. Enfermagem. Tolerância. Dependência.

\begin{abstract}
The use of clonazepam throughout Brazil has been a factor of concern in the public health area and in several other aspects, due to the large amount of dispensation. This study aims to discuss the clonazepam wholesale use and demonstrate the importance of nursing care. It is a documentary research with bibliographic basis provided by National Health Surveillance Agency (ANVISA) and a study conducted based in articles and bibliographies to be able to identify how nursing professionals can intervene to mitigated or solve the issue. The use of Clonazepam has been increasing in recent years, but despite its safety by its high therapeutic index, the low risk of toxicity and rare cases of overdose, if used for prolonged time can generate problems such as tolerance, dependence and even withdrawal crises during withdrawal of the drug. It is understood that the search for medicines that relieve the symptoms of mental health problems and the lack of sufficient information has generated an indiscriminate use, bringing the dependence as the main consequence. Therefore, it is believed that the nurse should have sufficient knowledge and preparation to act by decreasing the use of clonazepam, along with the multi professional team, because the knowledge about the action of this medicine as a cause of chemical dependence, as well as planning and strategies implementation, can contribute significantly by predicting and minimizing harmful effects.
\end{abstract}

Keywords: Clonazepam. Nursing. Tolerance. Dependency.

\title{
1 INTRODUÇÃO
}

O uso de medicamentos que causam alterações no sistema nervoso tornou-se crescente nas últimas décadas, podendo ou não produzir reações no corpo e na mente. Grande parte da população faz uso de algum psicotrópico e principalmente aqueles que são legais. $\mathrm{O}$ clonazepam está entre um dos mais prescritos no mundo, sendo que $10 \%$ da população teve contato com o medicamento por ano, com estimativas de que 1 a $3 \%$ já os tenha consumido regularmente por mais de um ano. (FORSAN, 2010; CARVALHO, 2017)

Essa maior procura por esse tipo de medicamento pode estar ligada a mudança dos hábitos de vida das populações, sendo associado ao alívio de ansiedade, estresse, dentre outros fatores. São vários os motivos que contribuem para o aumento do uso indiscriminado do clonazepam, desde a falta de conhecimentos dos riscos por parte do usuário até a falta de preparação dos profissionais no momento da prescrição e na orientação, aumentando assim o risco de desenvolver consequências graves pelo uso do medicamento. (AMARAL, 2012; 
Seu uso pode causar efeitos colaterais como sonolência, mas também pode levar a consequências mais graves como perda de memória e disfunções cognitivas, principalmente se usado de forma incorreta ou por tempo superior ao indicado. (CHELLAPPA, 2007)

Embora faça parte de uma classe segura de medicamentos, usuários de clonazepam nem sempre recebem a orientação necessária em relação aos riscos, como a dependência e a tolerância que se trata de um processo de resistência ao efeito do medicamento, onde mesmo dobrando a dose o usuário não tem o efeito esperado e dificulta a possível retirada do medicamento, quando se tem esses efeitos não é indicado interromper o uso, pois provavelmente levará a uma síndrome de abstinência, podendo causar danos à saúde. (CHELLAPPA, 2007). Portanto, a enfermagem pode atuar contribuindo para que esse cenário seja transformado, promovendo ações que evidenciem seus efeitos a longo prazo para conscientizar a população.

Este estudo trata-se de um estudo documental com fundamentação bibliográfica, composta por dados da dispensação do medicamento obtidos na anvisa, pesquisa em artigos e revistas cientificas relacionados à temática e associando à enfermagem. Objetivou-se discutir o uso indiscriminado de clonazepam e demonstrar a importância da assistência de enfermagem

\section{MÉTODOS}

Para atender ao objetivo proposto, a pesquisa se constituiu de um estudo documental com fundamentação bibliográfica, ou seja, os dados utilizados são secundários. Para a fundamentação teórica foi realizada uma revisão de literatura sobre a dinâmica e cinética do Clonazepam, o uso abusivo e seus riscos se usado de forma indiscriminada. com referência de dados on-line disponíveis nas bases de dados: Nescon (Núcleo de educação em Saúde Coletiva), SciELO (Scientific Eletronic Library Online), monografias, teses, dissertações, livros, anais de congresso e revistas eletrônicas. Primeiramente foram selecionadas as publicações condizentes ao estudo, analisadas e posteriormente elaborado o referencial teórico. Para a pesquisa documental, foram extraídos dados da anvisa, no Boletim de farmacoepidemiologia do Sistema Nacional de gerenciamento de produtos controlados (SNGPC), que fornece dados de dispensação de medicamentos controlados entre os anos de 2007 e 2010. Nestes sistemas de informação, constam dados de vários medicamentes, foram 
selecionados os que seriam usados, condensados e apresentados em forma de gráfico, também foram coletados dados em forma de gráfico retirados de um artigo publicado, no qual se trata de uma pesquisa realizada na cidade de Criciúma, SC, onde foram avaliados dados da utilização de Clonazepam entre 95 usuários. Para relacionar com a assistência de enfermagem foi realizada uma busca on-line com as seguintes palavras chaves: Saúde Mental, atenção primária à Saúde e medicalização na atenção básica, juntamente com informações e experiências adquiridas no decorrer do curso.

\section{FARMACODINÂMICA, FARMACOCINÉTICA E CONSEQUÊNCIAS DO USO INDISCRIMINADO DE CLONAZEPAM}

Clonazepam é um estimulante de GABA, um neurotransmissor inibidor neurossináptico, que tem sua atuação através do controle da abertura dos canais de cloro (Cl-), portanto, há entrada de carga negativa nas células e ocorre a diminuição da despolarização, inibindo assim as sinapses, são geralmente utilizados na forma de comprimidos pela via oral, mas há também apresentações injetáveis e líquidas. (LOPES, 2019; SILVA, 2003)

O medicamento possui como principal propriedade farmacológica inibição leve das funções do sistema nervoso central (SNC), portanto, permite o uso terapêutico como anticonvulsivante, sedação, relaxamento muscular e efeito ansiolítico. Clonazepam possui rápida absorção, com média de uma a quatro horas para obter máxima concentração plasmática, uma meia-vida longa de aproximadamente de 30 a 40 horas, $90 \%$ de biodisponibilidade, uma alta afinidade com proteínas plasmáticas com cerca de $82 \%$ a $86 \%$. É metabolizado pelas enzimas do citocromo P-450 e cerca de $70 \%$ eliminado na sua forma inativa através da filtração glomerular, os outros $30 \%$ são eliminados pelas fezes. (CLONAZEPAM BULA; ANDREATINI, 2001; MENDONÇA, 2005)

$\underline{\text { Seguindo a mesma linha de que se trata de um medicamento de meia vida longa, é }}$ possível compreender que permite uma maior concentração plasmática por mais tempo e que pode ser positivo em relação à descontinuidade, ou seja, pode-se tentar diminuir as doses gradualmente para evitar grandes prejuízos.

$\underline{\text { O clonazepam faz parte do grupo de drogas mais utilizadas, possivelmente por possuir }}$ uma boa tolerabilidade e por ter um rápido início de ação, mas problemas como o abuso do 
medicamento, dependência e tolerância não devem ser ignorados. (ANDREATINI, 2001) Compreende-se que essa boa tolerabilidade possa justificar o uso indiscriminado.

Entretanto, por se tratar de um depressor do SNC como dito anteriormente, torna-se necessário informar aos pacientes que fazem seu uso, sobre a realização de certas ocupações que exigem atenção, como dirigir veículos ou operar máquinas, já que requer uma maior habilidade e agilidade mental.

A segurança do clonazepam é comprovada por seu alto índice terapêutico, seu baixo risco de toxicidade, raros casos de overdose, casos esses que podem ser revertidos através de um eficaz antagonista, o flumazenil. (MENDONÇA, CARVALHO 2005). Contudo, mesmo que seja um fármaco relativamente seguro, não se deve usar por um tempo superior ao indicado devido aos possíveis riscos de efeitos colaterais a longo prazo.

Quando se usa clonazepam por um período prolongado pode levar ao desenvolvimento de dependência e tolerância, esse risco torna-se aumentado de acordo com o tempo de tratamento, a dose usada, em pessoas que fazem uso de drogas e álcool e as que possuem antecedentes de desenvolver dependência._(CLONAZEPAM BULA; MENDONÇA, CARVALHO, 2005; LEITE, 2015; KATZUNG, 1994). Portanto, esses indivíduos devem ser observados e acompanhados com cuidado, já que os riscos de desenvolver dependência pelo Clonazepam são mais altos.

Outra questão muito importante é em relação a descontinuidade do tratamento, pois não se pode parar com o medicamento de forma brusca, é necessário uma diminuição gradual da dose com cerca de $1 / 4$ da dose em uso a cada uma/duas semanas, quando se interrompe o tratamento sem antes reduzir a dose, leva a pessoa a ter grandes riscos de desenvolver a Síndrome de Abstinência, que causa sintomas como psicoses, distúrbio comportamental, tremor, sudorese, alucinações, agitação, dispneia, cefaleia, dores musculares (comumente a angina), extrema ansiedade, confusão e irritabilidade, alguns desses sintomas podem ser confundidos com a patologia de base ou até mesmo com outras. (SILVA, 2003; CLONAZEPAM BULA; MENDONÇA, CARVALHO, 2005; LEITE, 2015; FORTES, 2018). Relatos de "infarto" são bem comuns nas crises de abstinência, como foi dito a angina é comum e se associado à ansiedade, dispneia e sudorese, é provável que o indivíduo conclua que se trata de um Infarto Agudo do Miocárdio. 
A maior preocupação em relação ao Clonazepam se dá pelos efeitos a longo prazo MENDONÇA, CARVALHO, 2005), dito isso, o clonazepam merece uma atenção especial dos profissionais da saúde, não só por parte de médicos e farmacêuticos, mas também pelos profissionais de enfermagem que tem a função de orientar e alertar quanto aos efeitos que esses medicamentos podem causar se usados de maneira indevida, e também de toda a equipe $\underline{\text { multiprofissional promovendo uso racional do medicamento e colaborando para a diminuição }}$ das taxas de uso.

\section{DADOS DE DISPENSAÇÃO DO CLONAZEPAM}

O consumo de Clonazepam é controlado no Brasil através da prescrição e dispensação, que está incluída na Portaria SVS/MS 344, de 12 de maio de 1998, contudo, a venda proibida ainda é um fato que ocorre, ou seja, esse controle se torna falho em algumas situações, pois pacientes usam artifícios para que consigam o medicamento de forma indevida, seja através da indução de prescrições, adulteração de receitas, ou até mesmo acesso ao sistema farmacêutico. (BRASIL, 1998)

$\mathrm{O}$ que se pode julgar é que nem sempre o paciente tenha sido consultado por um médico psiquiatra ou a receita é prescrita por um médico de outra especialidade, o que favorece uma utilização inadequada e leva a um tratamento não eficaz do problema.

De acordo com a Anvisa, o clonazepam foi o ansiolítico mais usado no Brasil no ano de 2010, em relação ao bromazepan e alprazolam, sendo eles medicamentos pertencentes a mesma classe, o clonazepam foi dispensado em quantidades superiores ao dobro dos outros dois medicamentos citados. Sendo de clonazepam uma média de 258 unidades físicas dispensadas (UFD) por farmácia, média de 109 UFD de Bromazepan e média de 106 UFD de alprazolam. (GRÁFICO 1)

GRÁFICO 1. Comparação entre dados de dispensação dos ansiolíticos clonazepam, bromazepan e alprazolam no Brasil no ano de 2010. 


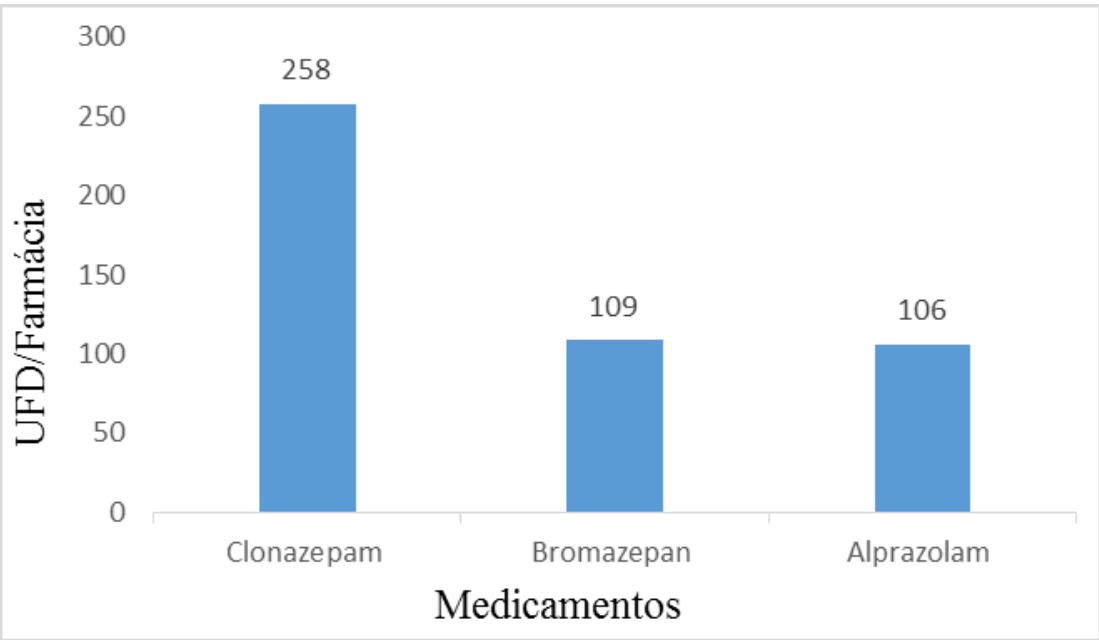

Fonte: SNGPC/Anvisa (2011)

O uso de clonazepam no Brasil nos anos de 2007 a 2010 aumentou em cerca de $3.685,71 \%$, de acordo com dados recolhidos do Boletim de farmacoepidemiologia do Sistema Nacional de gerenciamento de produtos controlados (SNGPC), no ano de 2007 teve uma média de 7 UFD por farmácia, 2008 de 176 UFD, 2009 de 214 UFD, já no ano de 2010 teve uma média de 258 UFD por farmácia, o que corresponde a 10,5 milhões de caixas de clonazepam. (GRÁFICO 2)

GRÁFICO 2. Dados de dispensação do clonazepam dos anos de 2007 a 2010 no Brasil.

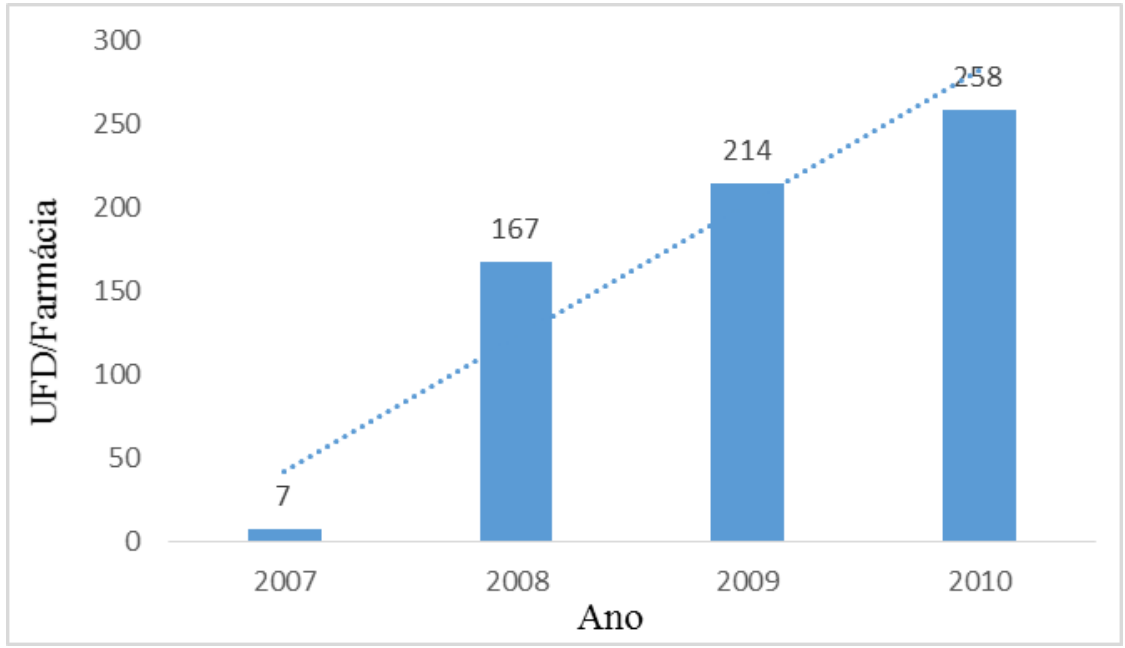

Fonte: SNGPC/Anvisa (2011) 
Uma pesquisa realizada com 95 usuários de benzodiazepínicos com idade superior a 18 anos, na rede pública de saúde do município de Criciúma, SC no ano de 2018, demonstra que a utilização do clonazepam é maior na faixa etária de 46 a 60 anos, sendo a maioria do sexo feminino com $31,6 \%$ de uso, seguido pelo sexo masculino com $15,8 \%$ de uso. O restante está distribuído pelas outras faixas etárias, sendo mais usado em pessoas acima de 60 anos do que as de 18 a 45 anos. (PERUCH, 2018)

Essa mesma pesquisa citada acima, demonstra que a utilização do clonazepam por tempo superior a 6 meses foi de 88,4\%. (GRÁFICO 3)

GRÁFICO 3. Prevalência do uso de Clonazepam com duração superior a seis meses entre 95 usuários entrevistados na rede pública de saúde do município de Criciúma, SC no ano de 2018.

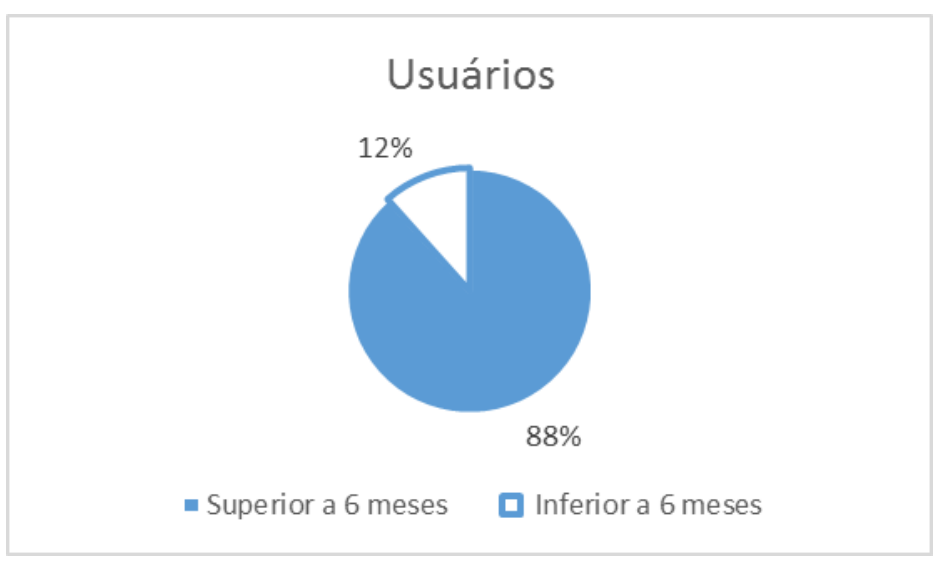

Fonte: PERUCH (2018)

Pesquisas recentes mostram que o consumo de clonazepam não diminuiu com o passar dos anos, apesar de sua dispensação ser controlada por medidas como a receita B - azul, cadastro pessoal e identificação do comprador com documento com foto. ((BRASIL, 1998)

Existe ainda uma média em que cada clínico teria por volta de 50 pacientes dependentes de clonazepam, e que destes, $50 \%$ desejam descontinuar o uso e $30 \%$ acreditam que os profissionais prescritores chegam inclusive a estimular o uso da medicação. (NASTASY, RIBEIRO, MARQUES, 2008)

Em uma pesquisa realizada em uma farmácia básica de Viçosa-MG, no mês de agosto do ano de 2015, demonstrou que houve um consumo de clonazepam de aproximadamente 
39.620 unidades físicas deste medicamento, sendo a população da cidade estimada para 2018 de 78.286 habitantes. (SANTOS, FRANCO, 2015)

Outra pesquisa realizada em uma drogaria do município Santa Inês- MA no período de maio a junho de 2018, demonstra que foram dispensadas cerca de 269 unidades físicas do clonazepam, tendo a cidade uma população estimada de 88.013 pessoas no ano de 2017 . (CAZAROTTI, LIMA, MIRANDA, SOUSA, BISPO, 2018). Mesmo sendo um número menor em relação a outra pesquisa, ainda é um número alto considerando que são resultados obtidos de apenas uma medicação da classe.

Já em outra pesquisa realizada em 2016 no Ambulatório Municipal de Saúde Mental do Município de Sorocaba, estado de São Paulo, onde são atendidas em média 4.600 pacientes ao mês, foram selecionados 330 deles para realização do estudo, o resultado foi que 167 fazem uso do clonazepam, sendo que apenas 73 tinham indicação de uso. (NALOTO, LOPES, BARBERATO FILHO, LOPES, DEL FIOL, BERGAMASCHI, 2016)

\section{ASSISTÊNCIA DE ENFERMAGEM}

Confere ao profissional de Enfermagem atribuições como, administrar, educar, cuidar e pesquisar, isso torna o enfermeiro indispensável em relação ao cuidado com o paciente, o respeitando como um ser bio-psico-social, uma pessoa com dignidade e características únicas. (BARRETO, 2014). Em função disso, o profissional de enfermagem pode buscar formas de prestar cuidados de qualidade, facilitando o processo de recuperação.

A importância da enfermagem durante o uso de clonazepam inclui a tomada de decisões e ações onde o mesmo, possa atuar. (BARRETO, 2014) Dito isso, o profissional deve elaborar propostas de intervenção já que é ele que passa maior parte do tempo com o paciente e possui acesso aos dados de todos os pacientes. Primeiramente, deve-se fazer um levantamento dos usuários de Clonazepam através da busca ativa, conhecer a realidade dessas pessoas, convidá-los para ser esclarecido sobre a medicação usada, a partir daí é possível implantar as propostas.

Contudo, a enfermagem não trabalha sozinha, precisa de toda uma equipe multiprofissional somando saberes para soluções efetivas, tratando o paciente com os princípios da integralidade e equidade, onde cada profissional atua em sua área, para que no 
final o paciente seja tratado como um todo. (PAULA, 2019). Como dito anteriormente, a enfermagem passa mais tempo com o paciente, conhece mais suas necessidades e pode até capacitar a equipe prescritora e dispensadora da medicação, visando diminuir as quantidades de prescrições e dispensações.

Nem sempre os pacientes são devidamente informados sobre o uso correto do Clonazepam, como a indicação, contraindicações, tempo do tratamento, cuidados no uso, empréstimo do medicamento através de outros usuários, pois existe uma falha na transmissão de informações. Isso se torna uma das principais causas do uso indiscriminado, o que diminui o êxito da terapêutica indicada. (FORTES, 2018; BRASIL, 2006; MENDES, 2015) Levando em consideração, é necessário identificar grupos que necessitam de informação e conscientizá-los sobre os riscos e benefícios do clonazepam, como também capacitar a equipe para que ela possa orientar os pacientes ou até mesmo conhecidos que façam uso do medicamento.

Existem também dificuldades ao se propor mudanças de condutas pré-estabelecidas, como a renovação automática de receitas sem reavaliação. (REIS, ALVES, CUNHA, CAVALLI, AGUIAR, 2018) Reconhecendo isso, o planejamento e execução de ações para minimizar os efeitos adversos do uso indiscriminado de devem prevalecer em prol da saúde dos pacientes.

A informação deve ser passada de forma simples, clara, sem julgamentos (BRASIL, 2006), pois cada indivíduo tem suas necessidades, levando em consideração o estado socioeconômico, cultural e emocional do paciente.

É importante também organizar palestras e reuniões, procurar formas de aquisição de recursos audiovisuais e panfletos, articulando seus recursos comunitários. (ROCHA, WERLANG, 2013; TADOKORO, 2012) O resultado esperado é a produção de um material educativo, que auxilie na capacitação de profissionais de enfermagem e conscientização de pacientes em tratamento com clonazepam, não só na atenção primária, mas em todos os diferentes serviços de saúde onde for observada a necessidade.

Recursos esses como por exemplo, os grupos de ajuda mútua, no qual pessoas que passam pela mesma situação, possam fornecer apoio na tentativa de diminuir a sensação de isolamento. Através da comunicação é possível aprender com outros pacientes e com os 
profissionais presentes, técnicas para que seja feito o controle do estresse e praticá-las como parte da rotina diária, tais técnicas podem ser essenciais para melhoria da qualidade de vida.

Para isso, o enfermeiro precisa conhecer os recursos comunitários para que os encaminhamentos sejam realizados de forma apropriada para investigação e tratamento corretos do uso indiscriminado de clonazepam. (VIDEBECK, 2012). Pois não adianta elaborar excelentes propostas e não ter os recursos comunitários necessários,

O enfermeiro como coordenador do setor deve saber liderar e manter a harmonia da equipe, (TEIXEIRA, SILVA, DRAGANOV, 2018) pois um ambiente tranquilo poderá contribuir para uma melhor assistência.

Resumindo, o profissional de enfermagem primeiramente precisa ter conhecimento sobre o medicamento e sobre a realidade desse consumo, como também sobre farmacologia, para que possa trabalhar alguns pontos principais que são necessários, como:

- Inicialmente é necessário definir as causas relatadas pelos pacientes para uso do medicamento, avaliar se há outras comorbidades presentes, como depressão, e se o paciente faz uso de outras medicações que possam interagir com o clonazepam. (MENDES, 2013; GAVIN, 2013) Essa busca pode ser feita através da anamnese e é importante para que seja possível intervir nos motivos do uso de Clonazepam e nos problemas citados a seguir.

- Quantidade de medicamento e duração do tratamento: Trata-se um dos aspectos mais importantes, pois parte daí as consequências e deve ser dada ênfase na orientação. É necessário informar os benefícios e as possíveis consequências do uso incorreto ou suspensão do clonazepam. (BRASIL, 1998) É possível conseguir isso através de agendamento de consultas periódicas para um melhor acompanhamento.

- Consequências do uso indiscriminado: É importante informar as consequências do não cumprimento do tratamento ou uso prolongado do clonazepam. (BRASIL, 1998) Como a síndrome de abstinência, tolerância e dependência, já citados anteriormente.

- Retirada do medicamento: Necessita-se ensinar e/ou reforçar à cada paciente como fazer o desmame medicamentoso de acordo com suas individualidades e 
das normas propostas pelo profissional prescritor. (LEITE, 2015; MARCOLAN, URASAKI, 1998) Como citado no início do trabalho, se a retirada do medicamento não ocorrer de forma gradual, há grandes chances de causar todos aqueles sintomas físicos e psíquicos relacionados à abstinência, por isso a importância de reforçar como deve ser feito o desmame.

- Outras opções de tratamento: São várias as indicações de uso do clonazepam, como transtornos do sono, ansiedade, depressão, transtornos de humor. (HAESER, 2006; BRASIL, 2013) Existe a possibilidade de procurar outros meios, como medidas não farmacológicas ou com outros fármacos que causem menos danos ao organismo, desde que com cautela, para que não se troque a dependência de um fármaco por outro, seguindo sempre a orientação do profissional que o prescreve.

Podem ser considerados pontos positivos para tentar chegar ao objetivo, como a criação de vínculo entre equipe de saúde e paciente, (TADOKORO, 2012; MENDES, 2013) ou seja, o enfermeiro pode demonstrar interesse, oferecer encorajamento para mudança, apoio, deixar claro que acredita que o paciente é capaz de mudar sua atual realidade, revisar a importância do acompanhamento com a equipe multiprofissional, principalmente com o psicólogo, no qual é possível falar sobre seus sentimentos e discutir técnicas comportamentais necessárias ao controle da ansiedade e dos outros motivos que o levaram a usar o Clonazepam.

O Clonazepam destina-se a aliviar sintomas, ou seja, não trata o problema (VIDEBECK, 2012). Portanto, os usuários do medicamento supracitado necessitam saber dessa informação para que não confiem que apenas o clonazepam irá sanar o problema de origem.

Como estratégia para diminuir a dependência e uso de clonazepam, quando o paciente é um idoso, pode-se propor a reinserção em grupos sociais, de atividade física se indicado e de artesanato, para que ele se sinta valorizado. (MARCOLAN, URASAKI, 1998; SILVA, VALENTE, BITENCOURT, BRITO, 2010; SILVA, OLIVEIRA, WANDERLEY, LIMA, TELES, 2015). E deve haver também um maior critério na escolha do tratamento, sendo avaliada sua função física, biológica e psicossocial. 
De acordo com a pesquisa realizada com usuários de benzodiazepínicos na rede pública de saúde do município de Criciúma-SC (PERUCH, 2018), percebe-se que há um predomínio da população feminina em uso do clonazepam, então faz-se necessário um foco nas estratégias que beneficiem essas mulheres.

A psicoeducação, que se trata basicamente de dar informações sobre o medicamento, apoio e alternativas possíveis, é sempre recomendado como uma forma de intervenção. (SPAGNOL, SANTIAGO, CAMPOS, BADARÓ, VIEIRA, SILVEIRA, 2010; LEMES, ONDERE NETO, 2017) É possível compreender que intervenções em todos os níveis de atenção, como distribuição de folhetos informativos, aconselhamento e capacitações podem facilitar a redução do uso de Clonazepam, estratégias psicológicas, como psicoterapia de grupo e terapia cognitivo-comportamental, podem também ser úteis.

\section{CONSIDERAÇÕES FINAIS}

Entende-se através deste estudo, que o uso de clonazepam está em um nível preocupante de acordo com as pesquisas utilizadas. A busca por medicamentos que aliviem os sintomas de problemas de saúde mental e a falta de informações suficientes tem gerado um uso indiscriminado, trazendo como principal consequência a dependência. Acredita-se que o enfermeiro deve ter conhecimento e preparo suficiente para atuar diminuindo o uso de clonazepam, junto com a esquipe multiprofissional.

É necessário um olhar minucioso pelos profissionais da saúde, fornecendo as orientações necessárias aos pacientes, principalmente quando se trata de substâncias relacionadas com o SNC. A atuação do enfermeiro na atenção à saúde é importante, seja no conhecimento sobre a medicação, como também na inserção de medidas de educação continuada para os pacientes que são usuários de clonazepam e na capacitação da equipe multiprofissional de saúde para dispor de uma preparação adequada.

Nesse sentido, um melhor conhecimento sobre a ação desse medicamento como causador de dependência química, bem como a participação do profissional de enfermagem, podem contribuir significativamente prevendo e minimizando efeitos danosos, desenvolvendo novas estratégias e programando ações preventivas e eficazes, fundamentadas no conhecimento científico, fornecendo informações sobre o modo correto de utilizar o 
medicamento, bem como os males que os mesmos podem acarretar para a saúde, trata-se de estratégias para minimizar o uso incorreto do medicamento, buscando a melhoria da qualidade de vida dos pacientes, com ênfase na segurança e bem-estar.

\section{REFERÊNCIAS}

Alvarenga J.M; Loiola Filho A.I; Giacomin K.C; Uchoa E; Firmo J.O.A. Uso de benzodiazepínicos entre idosos: o alívio de "jogar água no fogo", não pensar e dormir. Rev. Bras. Geriatr. Gerontol, Rio de Janeiro, v. 18, n. 2, p. 249-258, 2015. Disponível em: < http://www.scielo.br/pdf/rbgg/v18n2/1809-9823-rbgg-18-02-00249.pdf >Acesso em: 27 mar. 2019.

Amaral B.D.A; Machado K.L. Benzodiazepínicos: uso crônico e dependência. Monografia - Centro Universitário Filadélfia, Londrina, 2012. Disponível em: < http://web.unifil.br/pergamum/vinculos/000007/000007A8.pdf > Acesso em 24 mar. 2019.

Andreatini R; Boerngen-Lacerda R; Zorzetto Filho D. Tratamento farmacológico do transtorno de ansiedade generalizada: perspectivas futuras. Rev Bras Psiquiatr. [S. l.], v. 23, n. 4, p. 233-242, 2001. Disponível em: < http://www.scielo.br/pdf/rbp/v23n4/7172.pdf > Acesso em 29 mar 2019.

Barreto L.M.C.L.S. Cuidados de enfermagem ao paciente em uso de benzodiazepínicos. Monografia - Universidade Federal de Santa Catarina, Florianópolis, 2014. Disponível em: < https://repositorio.ufsc.br/xmlui/bitstream/handle/123456789/167461/LÍLIA\%20MARIA \%20CRISTINA\%20LIRA\%20DE\%20SÁ\%20BARRETO_APSICOSSOCIAL_TCC\%28 $1 \% 29$.pdf? sequence $=1 \&$ isAllowed=y $>$ Acesso em 24 mar 2019.

Boletim de Farmacoepidemiologia do SNGPC. Panorama dos dados do sistema nacional de gerenciamento de produtos controlados: um sistema para o monitoramento de medicamentos no Brasil. [S. l.], v. 2, n. 2, p. 1-9. 2011. Disponível em: < http://www.anvisa.gov.br/sngpc/boletins/2011/boletim_sngpc_2edatualizada.pdf $>$ Acesso em 15 mar 2019.

BRASIL. Ministério da Saúde / Portaria no 3.916/MS/GM, de 30 de outubro de 1998. Política nacional de medicamentos. Diário Oficial da República Federativa do Brasil, Brasília: Editora do Ministério da Saúde, 1998. Disponível em: < http://bvsms.saude.gov.br/bvs/saudelegis/gm/1998/prt3916_30_10_1998.html > Acesso em: 11 maio 2019.

BRASIL. Ministério da Saúde / Portaria SVS/MS n³44, de 12 de maio de 1998. Aprova o regulamento técnico sobre substâncias e medicamentos sujeitos a controle especial. Brasília: Diário Oficial da União da República Federativa do Brasil. Disponível em: < 
http://bvsms.saude.gov.br/bvs/saudelegis/svs/1998/prt0344_12_05_1998_rep.html > Acesso em 20 mar 2019.

BRASIL. Ministério da Saúde. Saúde mental. Brasília: Ministério da Saúde, 2013. (Cadernos de Atenção Básica, 32). Disponível em: < http://189.28.128.100/dab/docs/portaldab/publicacoes/caderno_34.pdf $>$ Acesso em 19 maio 2019.

BRASIL. Ministério da Saúde. Secretaria de Ciência, Tecnologia e Insumos Estratégicos. O trabalho dos agentes comunitários de saúde na promoção do uso correto de medicamentos. Brasília: Ministério da Saúde, 2006. Disponível em: < http://bvsms.saude.gov.br/bvs/publicacoes/trabalho_agentes_saude_promocao_medicame

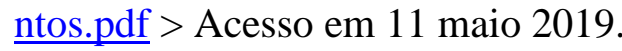

Carvalho C.G. Educação para saúde sobre o uso de benzodiazepínicos em um psf de um município mineiro. Monografia - Universidade Federal de Minas Gerais. Belo Horizonte, 2017. Disponível em: < https://www.nescon.medicina.ufmg.br/biblioteca/imagem/CEZAR-GONCALVESCARVALHO.pdf > Acesso em: 10 mar 2019.

Cazarotti M.L.B; Lima L.C; Miranda A.R; Sousa E.O; Bispo F.C.L. Psicotrópicos: Prescrições Médicas Dispensados em uma Drogaria no Município de Santa Inés - MA. Revista Eletrônica Acervo Cientifico. [S. l.], v. 2, e326, jan.2019. Disponível em: < https://www.acervomais.com.br/index.php/cientifico/article/view/326 > Acesso em 16 ago 2019.

Chellappa S.L. Sonolência excessiva diurna e depressão: causas, implicações clínicas e manejo terapêutico. Rev. psiquiatr. Rio Gd. Sul, Porto Alegre, v. 31, n. 3, 2009. Disponível em: < http://www.scielo.br/scielo.php?script=sci_arttext\&pid=S010181082009000400001 > Acesso em: 29 mar. 2019.

Clonazepam. [bula]. Domingos J. S. Iannotti. Lagoa de prata: Pharlab Indústria Farmacêutica S.A. Disponível em: < http://www.anvisa.gov.br/datavisa/fila_bula/frmVisualizarBula.asp?pNuTransacao=16250 162016\&pIdAnexo=3376913 > Acesso em 12 mar 2019.

Forsan, M.A. $\mathbf{O}$ uso indiscriminado de benzodiazepínicos: uma análise crítica das práticas de prescrição, dispensação e uso prolongado. 2010. Monografia - Universidade Federal de Minas Gerais, Campos Gerais, 2010. Disponível em: < https://www.nescon.medicina.ufmg.br/biblioteca/imagem/0649.pdf > Acesso em: 10 mar 2019

Fortes B.C. O uso de clonazepam no tratamento de zumbido em indivíduos em processo de envelhecimento: uma revisão sistemática. Dissertação - Universidade de passo fundo, Passo Fundo, 2018. [acesso em 2019 mar 25]. Disponível em: < 
http://tede.upf.br/jspui/bitstream/tede/1580/2/2018BibianaCallegaroFortes.pdf $>$ Acesso em 25 mar 2019.

Gavin R.S. Depressão, estresse e ansiedade: um enfoque sobre a saúde mental do trabalhador. Dissertação - Universidade de São Paulo, Ribeirão Preto, 2013. Disponível em: < http://www.teses.usp.br/teses/disponiveis/22/22131/tde-14012014-163411/pt-br.php $>$ Acesso em 22 maio 2019.

Haeser A.S. O estresse oxidativo e a depressão no Diabetes em modelo animal: O efeito do clonazepam. Dissertação - Universidade Federal do Rio Grande do Sul, Porto Alegre, 2006. Disponível em: < https://www.lume.ufrgs.br/bitstream/handle/10183/10451/000580910.pdf?sequence=1 > Acesso em 18 maio 2019.

Katzung B.G. Farmacologia básica e clínica. 5.ed. Rio de Janeiro: Guanabara Koogan S.A; 1994

Leite F.M. Diminuição do uso de benzodiazepínicos na penitenciária de Santa Vitória. Monografia - Universidade Federal de Minas Gerais, Santa Vitória, 2015. Disponível em: < https://www.nescon.medicina.ufmg.br/biblioteca/imagem/diminuicaouso-benzodiazepinicos-penitenciaria-vitoria.pdf > Acesso em 22 mar 2019.

Lemes C.B; Ondere Neto J. Aplicações da psicoeducação no contexto da saúde. Temas psicol. [S. l.], v. 35, n. 1, p. 1-28, 2017. Disponível em: < http://pepsic.bvsalud.org/pdf/tp/v25n1/v25n1a02.pdf > Acesso em 23 maio 2019.

Lopes M.A. Efeitos do clonazepam sobre as respostas defensivas medidas em ratos submetidos ao labirinto em T elevado. Dissertação - Universidade de São Paulo, Ribeirão Preto, 2010. Disponível em: < http://www.teses.usp.br/teses/disponiveis/17/17133/tde-03072010-224857/pt-br.php > Acesso em 25 mar 2019.

Marcolan J.F; Urasaki M.B.M. Orientações básicas para os enfermeiros na ministração de psicofármacos. Revista da escola de enfermagem da USP, [S. l.], v. 32, n. 3, p. 208-217, 1 out. 1998. Disponível em: <

http://www.scielo.br/scielo.php?script=sci_arttext\&pid=S008062341998000300004\&lng=pt\&tlng=pt > Acesso em: 20 maio 2019.

Marques T.F. Estratégias não medicamentosas para a abordagem dos usuários crônicos de ansiolíticos e antidepressivos. Monografia - Universidade Federal de Minas Gerais, Conselheiro Lafaiete, 2013. Disponível em: < https://www.nescon.medicina.ufmg.br/biblioteca/imagem/4618.pdf > Acesso em 10 maio 2019.

Mendes C.M.M. Estudo farmacoepidemiológico de uso e prescrição de benzodiazepínicos em Teresina. Tese - Universidade federal de do Ceará, Teresina, 
2015. Disponível em: <

http://www.repositorio.ufc.br/bitstream/riufc/13932/1/2015 tese_cmmmendes.pdf $>$

Acesso em 20 maio 2019.

Mendes K.C.C. O uso prolongado de benzodiazepínicos - uma revisão integrativa. Monografia - Universidade Federal de Minas Gerais, Pompéu, 2013. Disponível em: < https://www.nescon.medicina.ufmg.br/biblioteca/imagem/4077.pdf $>$ Acesso em 4 jun 2019.

Mendonça R; Carvalho A. O consumo de benzodiazepínicos por mulheres idosas. Revista eletrônica Saúde Mental Álcool e Drogas. [S. l.], v. 1, n. 2, p. 1-13, 2005. Disponível em: < http://www.revistas.usp.br/smad/article/view/38628/41475 > Acesso em 10 mar 2019.

Naloto D.C.C; Lopes F.C; Barberato Filho S; Lopes L;C; Del Fiol F.S; Bergamaschi C.C. Prescrição de benzodiazepínicos para adultos e idosos de um ambulatório de saúde mental. Ciênc. saúde coletiva. [S. l.], v. 21, n. 4, p. 1267-1276, 2016. Disponível em: < http://www.scielo.br/scielo.php?script=sci_arttext\&pid=S1413$\underline{81232016000401267 \& \operatorname{lng}=\mathrm{pt}}>$ Acesso em 16 ago 2019.

Nastasy H; Ribeiro M; Marques A.C.P.R. Abuso e Dependência dos Benzodiazepínicos. Rio de Janeiro: Associação Brasileira de Psiquiatria; 2008. Santos RN, Franco AJ. Consumo médio mensal de clonazepam em viçosa, disponibilizado pelo sus. Revista Científica Univiçosa. [S. l.], v. 7, n. 1, p. 459-464, 2015 Jan - dez. Disponível em: < https://academico.univicosa.com.br/revista/index.php/RevistaSimpac/article/download/57 $\underline{2 / 723}>$ Acesso em 16 ago 2019.

Paula R.A. Relação multiprofissional do trabalho em equipe na atenção básica de saúde. Monografia - Universidade Federal de Minas Gerais, São Sebastião do Paraíso, 2016. Disponível em: <

https://www.nescon.medicina.ufmg.br/biblioteca/imagem/0296.pdf $>$ Acesso em 10 maio 2019.

Peruch M.H. Perfil epidemiológico dos usuários e prevalência de uso crônico de benzodiazepínicos dispensados pelas farmácias públicas municipais de Criciúma-SC. Dissertação - Universidade Federal de Santa Catarina, Criciúma, 2018. Disponível em: < https://repositorio.ufsc.br/bitstream/handle/123456789/190160/PFMC-P0018D.pdf?sequence $=-1 \&$ isAllowed $=\mathrm{y}>$ Acesso em 24 maio 2019.

Reis I.L.F; Alves L.F.C; Cunha L.D.R; Cavalli M.A.P; Aguiar R.A.T. Renovação de prescrição médica na atenção primária: uma análise crítica. Revista Médica de Minas Gerais. [S. l.], v. 28, n. 1, 2018. Disponível em: < http://www.rmmg.org/artigo/detalhes/2360 > Acesso em 11 maio 2019.

Rocha B.S; Werlang M.C. Psicofármacos na Estratégia Saúde da Família: perfil de utilização, acesso e estratégias para a promoção do uso racional. Monografia - 
Universidade Federal de Pelotas; 2013. Disponível em: < https://www.scielosp.org/article/csc/2013.v18n11/3291-3300/ > Acesso em 8 maio 2019.

Santos R.N; Franco A.J. Consumo médio mensal de clonazepam em viçosa, disponibilizado pelo sus. Revista Científica Univiçosa. [S. l.], v. 7, n. 1, p. 459-464, 2015 Jan - dez. Disponível em: <

https://academico.univicosa.com.br/revista/index.php/RevistaSimpac/article/download/57 $\underline{2 / 723}>$ Acesso em 16 ago 2019.

Silva B.P; Oliveira D.G; Wanderley D.M.S; Lima R.S.C.L; Teles Y.C.F. Avaliação do uso de benzodiazepínicos por idosos. In: Anais do $4^{\circ}$ Congresso nacional de envelhecimento humano. p. 1-5, 2015; Paraíba. Paraíba: Universidade Federal da Paraíba; 2015. Disponível em: < http://www.editorarealize.com.br/revistas/cieh/trabalhos/TRABALHO_EV040_MD4_SA 3_ID3102_28082015000805.pdf $>$ Acesso em 4 jun 2019.

Silva C.M.C; Valente G.S.C; Bitencourt G.R; Brito L.N. A teoria do cuidado transpessoal na enfermagem: análise segundo Meleis. Rev Cogitare Enferm, [S. l.], v. 15, n. 3, p. 548551, Jul/Set 2010. Disponível em: <

https://revistas.ufpr.br/cogitare/article/viewFile/18902/12210 > Acesso em: 23 maio 2019.

Silva F.R. Efeitos de benzodiazepínicos sobre a atividade de neutrófilos de ratos avaliados por citometria de fluxo. Tese - Universidade de São Paulo, São Paulo, 2003. Disponível em: < http://www.teses.usp.br/teses/disponiveis/10/10133/tde-31052004153504/en.php $>$ Acesso em 25 mar 2019.

Spagnol C.A; Santiago G.R; Campos B.M.O; Badaró M.T.M; Vieira J.S; Silveira A.P.O. Situações de conflito vivenciadas no contexto hospitalar: a visão dos técnicos e auxiliares de enfermagem. Rev. esc. enferm. [S. l.], v. 44, n. 3, p. 1-9, 2010. Disponível em: < http://www.scielo.br/scielo.php?script=sci_arttext\&pid=S0080-

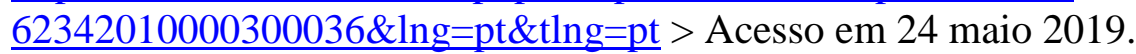

Tadokoro D.C. Transtornos mentais na atenção primária: uma reflexão sobre a necessidade de organizar e acolher a demanda dos usuários do SUS. Monografia Universidade Federal de Minas Gerais, Uberaba, 2012. Disponível em: < https://www.nescon.medicina.ufmg.br/biblioteca/imagem/3398.pdf > Acesso em 22 maio 2019.

Teixeira N.L; Silva M.M; Draganov P.B. Desafios do enfermeiro no gerenciamento de conflitos dentro da equipe de enfermagem. Rev. Adm. Saúde, [S. l.], v. 18 n. 73, 2018. Disponível em: 〈 http://www.cqh.org.br/ojs-2.4.8/index.php/ras/article/view/138/191 > Acesso em 24 maio 2019. 
Videbeck S.L. Enfermagem em saúde mental e psiquiatria. 5.ed. Porto Alegre: Artemed; 2012 